\title{
Attempted Validation of Surface Enhanced Laser Desorption Ionization- Time of Flight Derived Kinesin Biomarkers in Malignant Mesothelioma
} Stephen M. Brindley ${ }^{1,2 *}$, Brian C. Tooker ${ }^{1,2}$, Harvey I. Pass ${ }^{3}$ and Lee S. Newman ${ }^{1,2}$

${ }^{1}$ Department of Environmental and Occupational Health, Colorado School of Public Health, University of Colorado Denver, Aurora, CO, 80045, USA ${ }^{2}$ Division of Allergy and Clinical Immunology and Division of Pulmonary Science and Critical Care Medicine, Department of Medicine, University of Colorado Denver, Aurora, CO, 80045, USA

${ }^{3}$ Department of Cardiothoracic Surgery, New York University Langone Medical Center, New York, NY 10016, USA

\begin{abstract}
Surface-enhanced Laser Desorption/lonization Time-of-flight (SELDI-TOF) mass spectrometry allows researchers to search proteomes for biomarkers of disease. Validating SELDI-TOF results using traditional methodologies has yielded mixed results. Real-time PCR, ELISA and Western blot assays were utilized in an attempt to confirm the link between asbestos related cancers and kinesin family proteins, KIF5A and KIF18A. These proteins had been identified using SELDI-TOF and Classification and Regression Tree Analysis (CART) in serum of patients with asbestos related cancers. When well-characterized asbestos fibers were used to challenge mesothelial cell lines, mixed results in KIF5A and KIF18A RNA expression were observed. Western blot analysis comparing protein levels in mesothelioma cell lines versus transformed mesothelial cells yielded inconclusive results, as did Western blot analyses of human mesothelioma and non-cancerous peritoneum that had been obtained from the same patients. These results suggest that kinesin family proteins detected by SELDI-TOF CART are unlikely to be of use as biomarkers for malignant mesothelioma.
\end{abstract}

Keywords: SELDI-TOF; Validation; Mesothelioma; Biomarker; Asbestosis

Abbreviations: CART: Classification And Regression Tree; SELDI-TOF: Surface-enhanced Laser Desorption/Ionization Timeof-flight; KIF5A: Kinesin Heavy Chain Isoform 5A; KIF18A: Kinesinlike Protein 18A; MM: Malignant Mesothelioma; NIOSH: National Institute for Occupational Safety and Health; ELISA: Enzyme Linked Immunosorbent Assay

\section{Introduction}

Since Surface Enhanced Laser Desorption/Ionization Time-offlight (SELDI-TOF) mass spectrometry was first published in 1993 [1] it has been heralded as a powerful technique that allows researchers to search proteomes for biomarkers of disease. SELDI-TOF involves simplifying the proteome by separating it into various fractions that can then be bound to various chips, with disparate binding chemistries. Each chip/fraction combination can be analyzed using mass spectrometry to quantitate individual proteins. Proteins that are differentially expressed between control samples and disease samples are potential biomarkers.

Biomarker discovery can lead to earlier diagnosis that promises improved prognosis and potential targets for novel drug therapies $[2,3]$. SELDI-TOF has yielded biomarkers for some cancers and other disorders, and has been used to determine patients' response to therapy [4]. Although there are a few validated SELDI-TOF derived biomarkers [5-9], most have not been validated using more traditional approaches such as Enzyme Linked Immunosorbent Assay (ELISA) or Western Blot.

Malignant Mesothelioma (MM) is an aggressive cancer of the pleura and peritoneum that is caused by asbestos exposure. It is resistant to conventional therapies, and is often diagnosed only after it has reached an advanced stage [10]. In recent years, several studies have sought to define new biomarkers for early detection of MM [11-14].

In prior research, we identified Kinesin heavy chain isoform $5 \mathrm{~A}$ (KIF5A) and Kinesin-like protein 18A (KIF18A), motor proteins involved with intracellular positioning and trafficking of intracellular components, as potential biomarkers for progression to asbestos related cancer, such as lung cancer and malignant mesothelioma [11]. Our analysis utilized several protein biomarkers, including both KIF5A and KIF18A, to form a classification tree (CART) that could determine patient progression to asbestos related cancer, with $87 \%$ sensitivity and $70 \%$ specificity. The current study was undertaken to validate these SELDI-TOF derived biomarkers for asbestos-induced MM, using traditional approaches for biomarker discovery.

\section{Materials and Methods}

Our approach to establishing validity for these SELDI-TOF data was to test cell lines, human tissues, and sera for evidence of higher levels of KIF5A and lower levels of KIF18A, associated with asbestos related cancer. Our approach was tripartite. First, cell line studies were performed to determine if direct exposure of immortalized mesothelial cell lines to asbestos fibers in culture affected kinesin levels. Second, KIF5A and KIF18A levels in serum were evaluated by ELISA. Finally, Western blots allowed evaluation of KIF5A and KIF18A protein levels in both mesothelial cell lines and human tissues.

\section{Cell lines and tissue culture}

Met5A, K-562, MSTO-211H, H2452, H2052 and H28 cell lines were purchased from American Type Culture Collection (Manassas,

*Corresponding author: Stephen M. Brindley, Division of Allergy and Clinical Immunology and Division of Pulmonary Science and Critical Care Medicine Department of Medicine, University of Colorado Denver, Aurora, CO, 80045, USA, Tel: +1-303-724-7223; Fax: +1-303-724-7212; E-mail: Stephen.brindley@ucdenver.edu

Received November 27, 2011; Accepted December 13, 2012; Published December 20, 2012

Citation: Brindley SM, Tooker BC, Pass HI, Newman LS (2013) Attempted Validation of Surface Enhanced Laser Desorption Ionization-Time of Flight Derived Kinesin Biomarkers in Malignant Mesothelioma. J Data Mining Genomics Proteomics S1:002. doi:10.4172/2153-0602.S1-002

Copyright: (c) 2013 Brindley SM, et al. This is an open-access article distributed under the terms of the Creative Commons Attribution License, which permits unrestricted use, distribution, and reproduction in any medium, provided the original author and source are credited. 
Citation: Brindley SM, Tooker BC, Pass HI, Newman LS (2013) Attempted Validation of Surface Enhanced Laser Desorption lonization-Time of Flight Derived Kinesin Biomarkers in Malignant Mesothelioma. J Data Mining Genomics Proteomics S1:002. doi:10.4172/2153-0602.S1-002

Page 2 of 6

VA), while LP9 cells were already available in our laboratory. Met5A and LP9 are mesothelial cell lines immortalized using SV40 and Tert, respectively. MSTO-211H, H2452, H2052 and H28 are mesothelioma cell lines, and K-562 is a myelogenous leukemia line that has high KIF5A expression.

Met5A cells were cultured in Medium199 with Earle's BSS, 0.75 $\mathrm{mM}$ L-glutamine and $1.25 \mathrm{~g} / \mathrm{L}$ sodium bicarbonate (ATCC, Manassas, VA), supplemented with $3.3 \mathrm{nM}$ Epidermal Growth Factor (EGF), $400 \mathrm{nM}$ hydrocortisone, $870 \mathrm{nM}$ insulin (BD, Bedford, MA), $20 \mathrm{mM}$ HEPES, $100 \mathrm{U} / 100 \mu \mathrm{g} / \mathrm{ml} / 0.292 \mu \mathrm{mg} / \mathrm{ml}$ penicillin/streptomycin/ glutamine, 1\% Fungizone (Thermo Scientific, Rockford, IL), 10\% fetal bovine serum (Sigma Aldrich, St. Louis MO), and 1X trace elements (MP Biomedicals, Santa Ana, CA). K-562 were grown in IMDM (Life Technologies, Grand Island, NY) $10 \%$ complete media with $1 \%$ Fungizone, MSTO-211H, H2452, H2052 and H28 were all grown in RPMI-1640 (Life Technologies, Grand Island, NY) $10 \%$ complete media, with 1\% Fungizone and LP9 cells grown in DMEM (Life Technologies, Grand Island, NY) 10\% complete media with 1\% Fungizone. All cells were cultured at $37^{\circ} \mathrm{C}$ with $5 \% \mathrm{CO}_{2}$.

\section{Human population samples}

Human serum samples from 30 asbestos-exposed mesothelioma cases and 30 asbestos-exposed non-cancer referents were obtained through the National Mesothelioma Virtual Bank. Also obtained from the Virtual Bank were paired mesothelioma and unaffected peritoneum from the 30 mesothelioma patients. Demographic information for study participants is shown in table 1 .

\section{Cell culture asbestos exposure}

Asbestos samples were a generous gift from the U.S. Geological Survey Crustal Geophysics and Geochemistry Science Center's Office of Environmental and Human Health (Denver, CO). White quartz
(99\% $\left.\mathrm{SiO}_{2}, 0.5-10 \mu \mathrm{m}\right)$ was purchased from Sigma-Aldrich (Saint Louis, MO). The samples were sterilized overnight in ultraviolet light, and then minerals were suspended in 1X Hanks Balanced Salt Solution (HBSS) at $1 \mathrm{mg} / \mathrm{ml}$. Samples were sonicated for 15 minutes in a room temperature $40 \mathrm{kHz}$ water bath sonicator (Branson, Danbury, CT), and aspirated 5X through 22-guage needles. Suspensions of NIOSH crocidolite standard 210-75-0043, NIOSH chrysotile standard 210-750043, and small particle control $\mathrm{SiO}_{2}$ were added to LP9 and Met5A cells to achieve concentrations of $10 \mu \mathrm{g} / \mathrm{cm}^{2}$, while HBSS was used as a negative control. Experiments were performed in quadruplicate and harvested at 4, 8 and 24 hours. When collected, cells were washed $2 \mathrm{X}$ in PBS, pH 7.4, to remove non-incorporated stimuli.

\section{RNA isolation and real time PCR}

RNeasy kits were used to isolate RNA per manufacturer's instructions (Qiagen, Valencia, CA). Following isolation, RNA was treated with DNAse to remove possible genomic DNA contamination. This DNA-free RNA was then used as a template for reverse transcription of cDNA, using Superscript II reverse transcriptase (Life Technologies, Grand Island, NY). This cDNA was then used as a template for realtime PCR amplification with primers for $\beta$-actin (Realtimeprimers. com, Elkins Park, PA), KIF5A and KIF18A (Origene, Rockville, MD). Primer sequences are shown in table 2.

Real-time PCR amplifications were performed using an iCycler iQ real-time PCR detection system (Bio-Rad, Hercules, CA). iQ ${ }^{\text {TM }}$ SYBR $^{\mathbb{R}}$ Green Supermix (Bio-Rad) was used to detect DNA amplification. Amplification conditions were as follows: Step one is $95^{\circ} \mathrm{C}$ for 3 minutes; Step two is 40 cycles of $95^{\circ} \mathrm{C}$ for $15 \mathrm{sec}, 58^{\circ} \mathrm{C}$ for $30 \mathrm{sec}$ and $72^{\circ} \mathrm{C}$ for $30 \mathrm{sec}$; Step three is $95^{\circ} \mathrm{C}$ for 1 minute and held at $4^{\circ} \mathrm{C}$.

\section{Enzyme-Linked Immunosorbent Assay (ELISA)}

ELISA kits for KIF5A and KIF18A were purchased from USCN

\begin{tabular}{|c|c|c|}
\hline & Tissues for Western Blot Analysis & ELISA Serum Samples \\
\hline \multicolumn{3}{|l|}{ Age } \\
\hline Mean Years (SD) & $65.9(8.80)$ & $61.5(9.18)$ \\
\hline \multicolumn{3}{|l|}{ Gender n, (\%) } \\
\hline Male & $25(83.33)$ & $9(90.00)$ \\
\hline Female & $5(16.67)$ & $1(10.00)$ \\
\hline \multicolumn{3}{|l|}{ Race $n,(\%)$} \\
\hline Caucasian & $28(93.33)$ & No information \\
\hline Hispanic & $2(6.67)$ & No information \\
\hline \multicolumn{3}{|l|}{ Smoking Status (\%) } \\
\hline Never Smoker & $14(46.67)$ & $5(50.00)$ \\
\hline Former Smoker & $15(50.00)$ & $5(50.00)$ \\
\hline Current Smoker & $1(3.33)$ & $0(0.000$ \\
\hline \multicolumn{3}{|l|}{ Mesothelioma } \\
\hline Yes & $30(100)$ & $4(40.00)$ \\
\hline No & $0(100)$ & \\
\hline Average Mesothelioma Stage (SD) & $2.77(0.43)$ & $2.25(0.50)$ \\
\hline \multicolumn{3}{|l|}{ Mesothelioma Histology (\%) } \\
\hline Epithelial & $21(70.00)$ & $3(75.00)$ \\
\hline Biphasic & $9(30.00)$ & $1(25.00)$ \\
\hline
\end{tabular}

Table 1: Patient demographics.

\begin{tabular}{|l|l|}
\hline Transcipt & Forward Primer \\
\hline KIF5A & 5'AGGAGAAGAGCCAGCAGAACCA \\
\hline KIF18A & 5'CTTGGACCAGTTCAGCCTATTCC \\
\hline B-Actin & 5'GGACTTCGAGCAAGAGATGG \\
\hline
\end{tabular}

\begin{tabular}{|l|}
\hline Reverse Primer \\
5'CGTTTTCGCTGGTGTCCACTGA \\
5'GCACACTTTGAGATGGTGGAGAC \\
5'AGCACTGTGTTGGCGTACAG \\
\hline
\end{tabular}

Table 2: Primer list. 
Citation: Brindley SM, Tooker BC, Pass HI, Newman LS (2013) Attempted Validation of Surface Enhanced Laser Desorption lonization-Time of Flight Derived Kinesin Biomarkers in Malignant Mesothelioma. J Data Mining Genomics Proteomics S1:002. doi:10.4172/2153-0602.S1-002

Life Science Inc. (Wuhan, China). ELISA's were performed per kit instructions. Briefly, standards, blanks and samples were diluted 1:10 in PBS $\mathrm{pH}$ 7.4, and added to plates pre-coated with either KIF5A or KIF18A antibody for 2 hour incubation at $37^{\circ} \mathrm{C}$. After removing supernatants from the wells, $100 \mu$ l Detection Reagent A was added and plates incubated for one hour at $37^{\circ} \mathrm{C}$. Plates were washed $3 \mathrm{X}$ in kit provided wash buffer and then $100 \mu \mathrm{l}$ of Detection Reagent B working solution was added to each well for 30 minutes incubation at $37^{\circ} \mathrm{C}$. Plates were washed $5 \mathrm{X}$, and $90 \mu \mathrm{l} 3,3^{\prime}, 5,5^{\prime}$ Tetramethylbenzidine (TMB) substrate added for 20 minute incubation at room temperature, followed by the addition of $50 \mu \mathrm{l}$ of stop solution. Finally, optical density readings were obtained at $450 \mathrm{~nm}$ on a kinetic microplate reader (Molecular Devices, Sunnyvale, CA). All samples were run in duplicate.

\section{Protein isolation and western blot analysis}

Cultured cells were centrifuged for 5 minutes at $400 \times \mathrm{g}$ to pellet cells, while tissue samples were frozen in liquid nitrogen and pulverized with a Bessman tissue pulverizer (Spectrum Labs, Rancho
Dominquez, CA). Next, cell pellets and pulverized tissue samples were both lysed in Tris-Triton buffer (10 mM Tris, $\mathrm{pH} 7.4,100 \mathrm{mM} \mathrm{NaCl}, 1$ mM EDTA, 1 mM EGTA, 1\% Triton X-100, 10\% glycerol, 0.1\% SDS, $0.5 \%$ deoxycholate) for 30 minutes at $4^{\circ} \mathrm{C}$. Protein concentrations were determined by the Coomassie protein assay (Pierce, Rockford, IL). In triplicate, equal amounts of protein were separated by Sodium Dodecyl Sulfate-Polyacrylamide Gel Electrophoresis (SDS-PAGE) on 7.5\% Mini-PROTEAN ${ }^{\circledR}$ TGX $^{\text {TM }}$ Precast Gels (Bio-Rad), using laemmli loading buffer. Proteins were transferred to a Polyvinylidene Fluoride (PVDF) membrane at $12 \mathrm{~V}$ for 40 minutes, for Western blot analysis using the Genie Blotter (Idea Scientific, Minneapolis, MN), and blocked for 1 hour in PBS with $0.05 \%$ Tween 20 and 5\% dry milk.

Antibodies used for Western Blot analysis were diluted in the blocking solution as follows: 1:2000 rabbit anti-KIF5A (Novus Biologicals, Littleton, CO), 1:1500 rabbit anti-KIF18A (Bethyl Laboratories, Montgomery, TX), 1:20,000 monoclonal anti- $\beta$-actinperoxidase (Sigma Aldrich, St. Louis MO), and 1:2,000 goat anti-rabbit IgG-HRP (Sigma-Aldrich, Saint Louis, MO). Protein bands were visualized using SuperSignal West Pico Chemiluminescent substrate
A. KIF5A Relative Expression in Met5A Cells

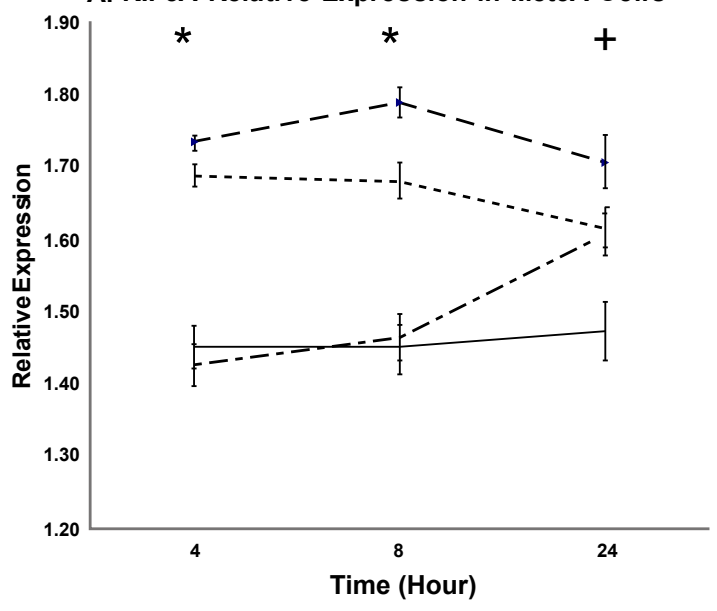

C. KIF18A Relative Expression In Met5A Cells

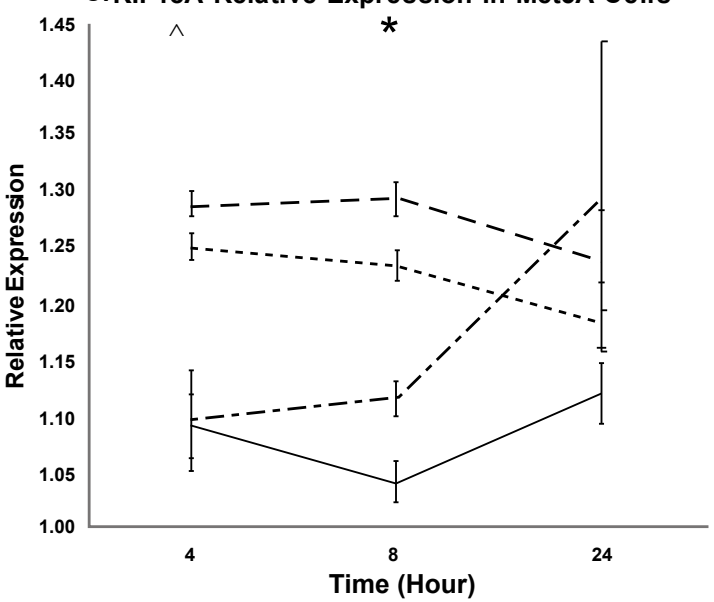

B. KIF5A Relative Expression In LP9 Cells

\#

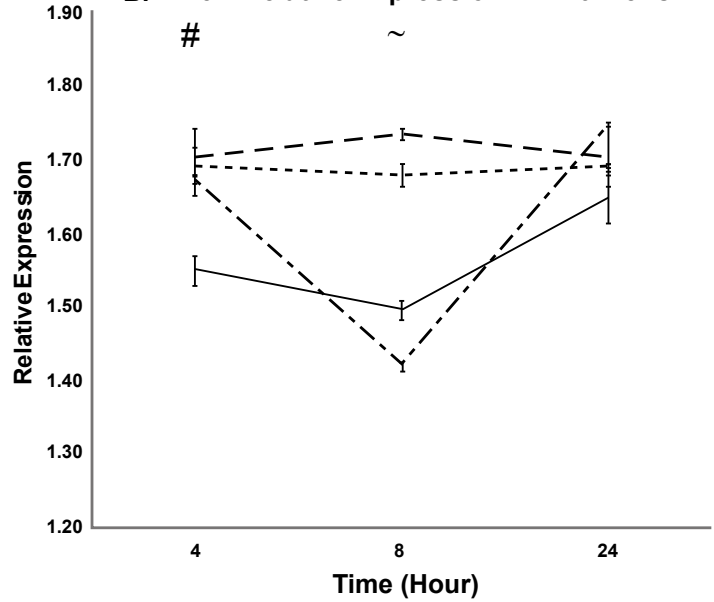

D. KIF18A Relative Expression In LP9 Cells

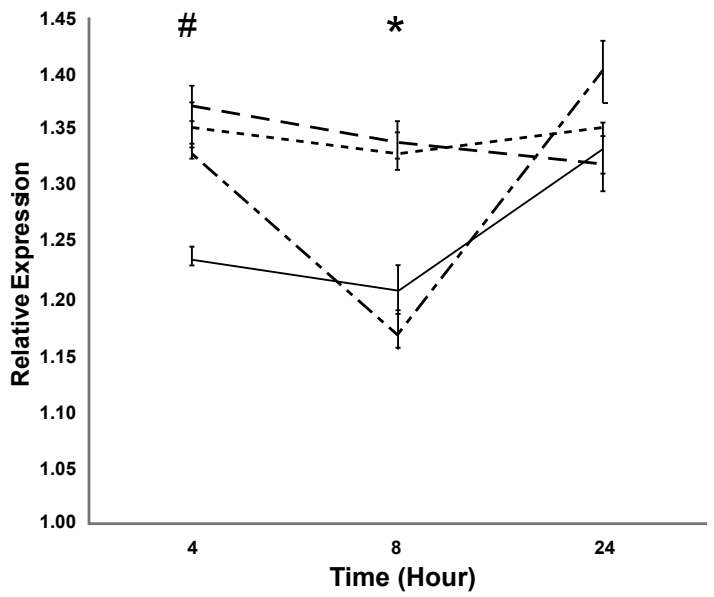

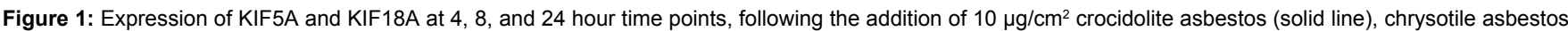
(dotted line), silicon dioxide (dashed-dotted line), or Hanks Balanced Salt Solution (dashed line). A Bonferroni adjusted $p$-value of $p \leq 0.01$ was considered significant, within each time point. Levels of significance are denoted by the following; $\left(^{*}\right)$ : crocidolite and $\mathrm{SiO}_{2}$ induced greater kinesin expression than $\mathrm{HBSS}$ or chrysotile, $(+)$ : crocidolite induced greater kinesin expression than HBSS, (\#): crocidolite induced greater kinesin expression than $\mathrm{HBSS}$ chrysotile and SiO ${ }_{2},(\sim)$ : crocidolite and $\mathrm{SiO} 2$ induced greater kinesin expression than $\mathrm{HBSS}$ or chrysotile and $\mathrm{SiO}_{2}$ induces greater expression than crocidolite, $\left({ }^{\wedge}\right)$ crocidolite induced greater kinesin expression than $\mathrm{HBSS}$ or chrysotile, while only $\mathrm{SiO}_{2}$ induced higher expression than HBSS. 

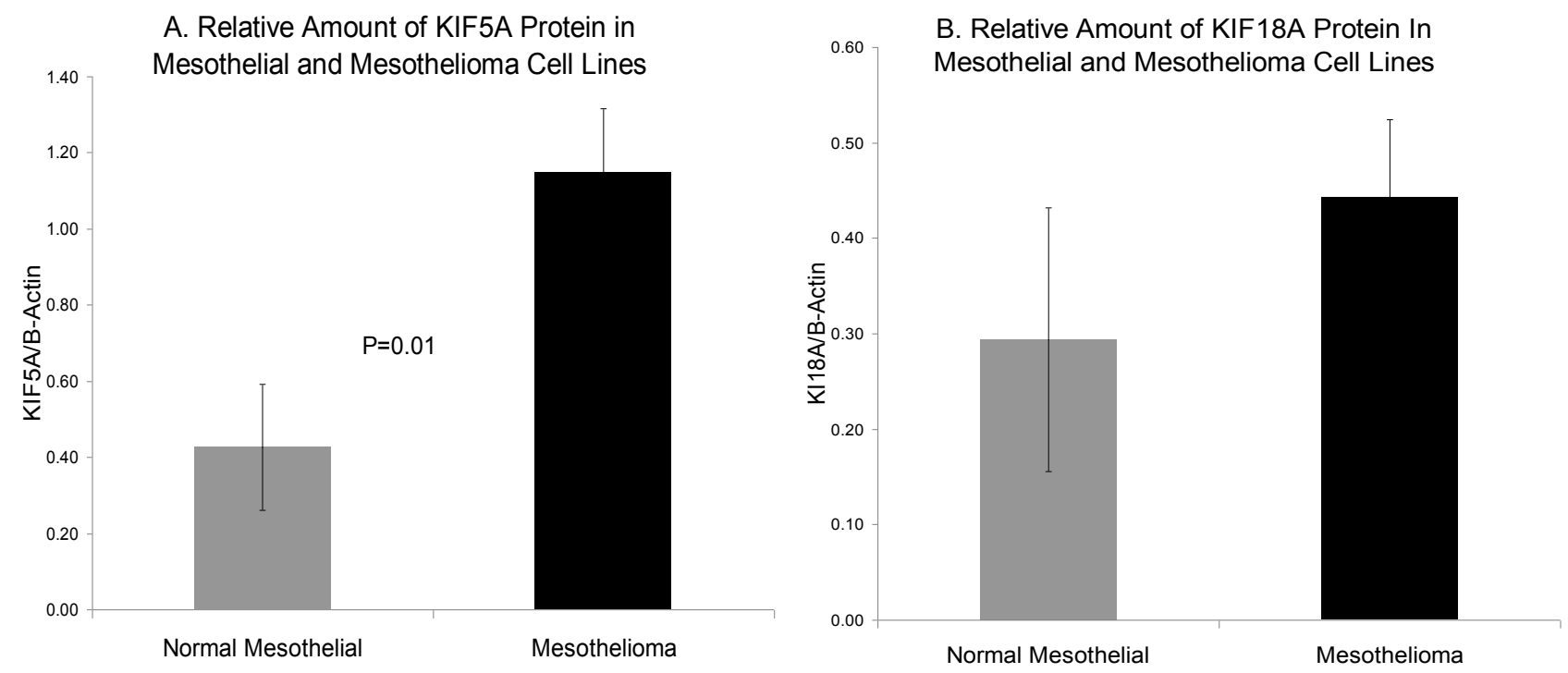

Figure 2: Relative expression of KIF5A (A) and KIF18A (B) in normal mesothelial (grey) and mesothelioma (black) cell lines. Protein levels were normalized using $\beta$-actin.

(Thermo Scientific, Rockford, IL). Protein amounts in the KIF5A, KIF18A and $\beta$-actin bands were quantified using Image software (NIH, Bethesda, Maryland). Amounts of $\beta$-actin protein were used to normalize KIF5A and KIF18A levels between blots. For human experiments, 30 mesothelioma tissue lysates were compared with 30 matched peritoneal lysates.

\section{Results}

\section{Kinesin mRNA expression in transformed mesothelial cells}

Transformed mesothelial cell lines were cultured and exposed to asbestos or controls to observe KIF5A and KIF18A mRNA expression at various time-points. As shown in figure 1, chrysotile asbestos produced no significant change in KIF5A or KIF18A, in either cell line at any of the time points. At the 4-hour time point, in MET5A cells, crocidolite induced higher expression of KIF5A $(\mathrm{p}<0.0005)$ and KIF18A $(\mathrm{p}<0.002)$, than did chrysotile (Bonferroni correction for significance at $\mathrm{p}<0.01$ ). At the 8-hour time point, crocidolite induced higher expression of KIF5A and KIF18A than did chrysotile (KIF5A p $<0.0005$ : KIF18A $\mathrm{p}<0.002$ ), or HBSS (KIF5A $\mathrm{p}<0.0002$ : KIF18A $\mathrm{p}<0.00004$ ). At 24 hours, crocidolite produced higher KIF5A expression only when compared to HBSS $(\mathrm{p}<0.007)$.

At the 4-hour time point, in LP9 cells, crocidolite induced higher expression of KIF5A and KIF18A, as compared to chrysotile (KIF5A $\mathrm{p}<0.005$ : KIF18A $\mathrm{p}<0.002$ ) and HBSS (KIF5A $\mathrm{p}<0.007$ : KIF18A $\mathrm{p}<0.0003)$. At the 8 -hour time points, crocidolite caused higher expression of KIF5A and KIF18A than did chrysotile (KIF5A p $<0.0002$ : KIF18A $p<0.005$ ), or HBSS (KIF5A $p<0.000004$ : KIF18A $p<0.003$ ), and lower KIF5A expression, when compared to $\mathrm{SiO}_{2}(\mathrm{p}<0.0006)$.

\section{ELISA}

To confirm SELDI-TOF results of higher KIF5A and lower KIF18A levels in the serum of patients with asbestos-related cancer, serum from mesothelioma patients and non-cancer asbestos exposed control subjects were evaluated using commercially available ELISAs. Although the standards and a cell lysate from a known kinesin- containing cell line showed positive results, the ELISAs failed to detect KIF5A or KIF18A in our sample sera, indicating that levels of KIF5A and KIF18A in these samples were all below the detection threshold for the kit $(3.12 \mathrm{ng} / \mathrm{ml})$.

\section{Cell line western blots}

Lysates from H2452, H2052, H28 and MSTO-211H mesothelioma cell lines were run side by side with the LP9 and Met5A mesothelial cell lines, to determine if KIF5A and KIF18A protein levels differ in cancer versus non-cancer cell lines. As illustrated in figure 2a, KIF5A analysis revealed significantly higher levels of KIF5A in the mesothelioma cell lines $(\mathrm{p}=0.01)$. Figure $2 \mathrm{~b}$ shows non-significant increases in KIF18A levels in the mesothelioma cell lines $(\mathrm{p}=0.28)$.

\section{Human tissue sample western blot}

Mesothelioma sample lysates $(n=30)$ were tested with matched peritoneal lysates to determine if differences in KIF5A levels observed in vitro could also be observed in tissue samples. Eight matched sets of samples were excluded from analysis due to extremely low levels of $\beta$-actin, which precluded proper analysis. As shown in figure 3, normalized mean KIF5A levels for the remaining mesothelioma samples were 0.63 ( \pm SEM 0.10), while normalized KIF5A levels in peritoneal samples were 2.60 ( \pm SEM 0.57), demonstrating KIF5A to be significantly higher $(\mathrm{p}<0.0014)$ in the peritoneal tissue samples, contrary to our prior hypothesis.

\section{Discussion}

The main goal of this study was to validate previously published SELDI-TOF data that had implicated KIF5A and KIF18A as candidate biomarkers of asbestos-related cancer. Our approach to establishing validity of the SELDI-TOF data was to test cell lines, human tissues and serum for evidence indicating higher KIF5A and lower KIF18A levels, associated with asbestos related cancer. We believe this multifaceted approach to testing the relevance of these SELDI-TOF derived biomarkers offers advantages as a strategy for validating SELDI-TOF data. Our attempts produced, at best, mixed results, and failed to 


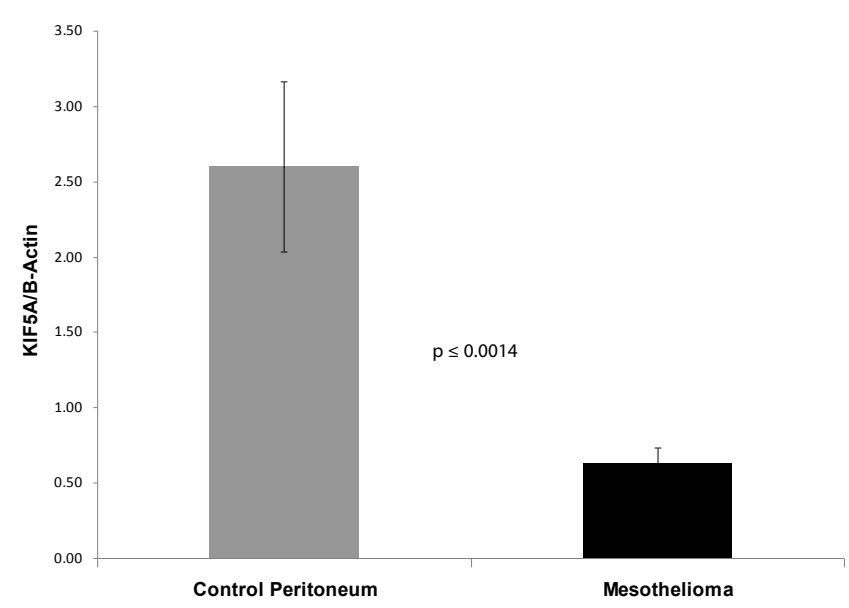

Figure 3: Relative expression of KIF5A in mesothelioma tissue (black) and paired peritoneal tissue (grey) from mesothelioma patients. Protein levels were normalized using $\beta$-actin.

demonstrate a consistent relationship between either KIF5A or KIF18A and mesothelioma using standard, non-SELDI-TOF-based methods.

There are a number of potential limitations to our study than may explain the inability to confirm SELDI-TOF CART analysis results. Initially, we considered using specimens from the same patients we had studied in the SELDI-TOF experiments. However, these types of samples were not readily available from that cohort, additionally, that cohort had been a mixed population of asbestos-induced cancers (lung cancer and mesothelioma). Therefore, the decision was made to examine KIF5A and KIF18A levels in mesothelioma tissue from a new cohort, and also employ immortalized mesothelium cell lines.

Cell culture asbestos experiments showed that crocidolite and chrysotile asbestos exposure failed to induce significant changes, in either KIF5A or KIF18A mRNA levels, when compared to the mineral control $\mathrm{SiO}_{2}$. One possible reason for these results may be related to when the asbestos was added to the cells. It was added once the cell lines had formed a confluent monolayer. It may have been better to add the particulates when the cells were $\sim 60 \%$ confluent and actively dividing. These growing cells may be more likely to take up particulate matter [15].

Western blot analysis of the cell lines demonstrated higher KIF5A protein levels in mesothelioma cell lines. This may indicate that KIF5A could be a valid marker for mesothelioma. Unfortunately, blots of the human biopsy samples produced results that were opposite to those expected for KIF5A protein, based on our SELDI-TOF CART data.

There is extensive literature questioning the value of cell lines in biomarker research [16]. In vitro microenvironments are considerably different from in vivo environments, and may account for the disparity in the results obtained in these two experiments. Another factor involves the controls used in these two experiments. The Met5A and LP9 cell lines used as controls in the cell line experiment produced very little KIF5A, while the peritoneal samples used as human controls yielded relatively large amounts of KIF5A. To help address these limitations, we used well-characterized asbestos fibers, and studied not only cell lines, but human specimens as well.

In our hands, commercially available kits were not sensitive enough to detect either KIF5A or KIF18A levels in serum samples, and therefore may have missed a small, but possibly significant difference between groups. Other studies have used matched patient serum and tissue to test for SELDI-TOF biomarkers, although not for KIF5A or KIF18A. In these studies, SELDI-TOF biomarkers were shown to differ between tissues and serum [17]. As such, it is unlikely that KIF5A and KIF18A would be helpful as serum biomarkers for progression to asbestos-related cancer.

The failure to confirm earlier SELDI-TOF findings in mesothelioma do not exclude the possibility relevance of kinesins in all forms of asbestos-induced lung cancer. In our SELDI-TOF study, we examined a Finnish cohort that included serum from 26 lung cancer patients and from 4 mesothelioma patients. For the current study, we elected to examine only mesothelioma cases from a U.S. registry. Thus, while we can draw conclusions about mesothelioma from this study, our data cannot rule out KIF5A and KIF18A as biomarkers for lung cancer.

SELDI-TOF's usefulness for biomarker discovery has been questioned for a number of reasons. There are instances in which investigators have not been able to reproduce results using different data sets [18-20], as was the case in our study; where different investigators detect unexpected biomarkers for the same disease [21-23], where there is failure to validate biomarkers using traditional methodologies [8], as in our study; and where validation studies detect the same biomarkers, but produce opposite results [24], as was suggested by some of our findings. Not surprisingly, most biomarkers lack sufficient disease specificity to work as stand-alone biomarkers [25,26]. Our data lend additional concern about the biological relevance of SELDI-TOF CART derived markers.

In conclusion, conventional methods for biomarker analysis did not confirm SELDI-TOF results in a second cohort of patients with asbestos-induced mesothelioma. Our data do not rule out the possibility of future confirmation in patients with asbestos-related lung cancer, but call into question the usefulness of KIF5A and KIF18A as overarching biomarkers of asbestos-related cancer.

\section{Acknowledgements}

We would like to thank our colleagues Dr. Geoff Plumlee and Gregory Meeke from the USGS for providing asbestos samples for this set of experiments. We would also like to thank the following funding sources; The Mesothelioma Virtual Bank for Translational Research, CDC-5 U19 OH (Dr. Pass, Co-Investigator) and the Mesothelioma Biomarker Discovery Laboratory, Early Detection Research Network (NCI/NIH) U01 5U01CA111295-05 (Dr. Pass, Principle Investigator) Finally, we would like to thank the patients who donated samples to the National Mesothelioma Virtual Bank, and thank the bank for providing the samples for these studies.

\section{References}

1. Hutchens T, Yip T (1993) New desorption strategies for the mass spectrometric analysis of macromolecules. Rapid Commun Mass Spectrom 7: 576-580.

2. De Bock M, de Seny D, Meuwis MA, Chapelle JP, Louis E, et al. (2010) Challenges for biomarker discovery in body fluids using SELDI-TOF-MS. J Biomed Biotechnol.

3. Liotta LA, Ferrari M, Petricoin E (2003) Clinical proteomics: written in blood Nature 425: 905

4. Weston AD, Hood L. (2004) Systems biology, proteomics, and the future of health care: toward predictive, preventative, and personalized medicine. J Proteome Res 3: 179-196.

5. Song Z, Dong R, Fan Y, Zheng S (2012) Identification of serum protein biomarkers in biliary atresia by mass spectrometry and enzyme-linked immunosorbent assay. J Pediatr Gastroenterol Nutr 55: 370-375.

6. Wang Q, Li D, Zhang W, Tang B, Li QQ, et al. (2011) Evaluation of proteomicsidentified CCL18 and CXCL1 as circulating tumor markers for differentia diagnosis between ovarian carcinomas and benign pelvic masses. Int $\mathrm{J}$ Bio Markers 26: 262-273. 
Citation: Brindley SM, Tooker BC, Pass HI, Newman LS (2013) Attempted Validation of Surface Enhanced Laser Desorption lonization-Time of Flight Derived Kinesin Biomarkers in Malignant Mesothelioma. J Data Mining Genomics Proteomics S1:002. doi:10.4172/2153-0602.S1-002

7. Xue A, Scarlett CJ, Chung L, Butturini G, Scarpa A, et al. (2010) Discovery of serum biomarkers for pancreatic adenocarcinoma using proteomic analysis. $\mathrm{Br}$ J Cancer 103: 391-400.

8. Walter M, Heinze C, Steiner T, Pilchowski R, von Eggeling F, et al. (2010) Imunochemotherapy-associated protein patterns in tumour tissue and serum of patients with metastatic renal cell carcinoma. Arch Physiol Biochem 116: 197-207.

9. O'Riordan A, Johnston O, McMorrow T, Wynne K, Maguire P, et al. (2008) Identification of apolipoprotein $\mathrm{Al}$ as a serum biomarker of chronic kidney disease in liver transplant recipients, using proteomic techniques. Proteomics Clin Appl 2: 1338-1348.

10. Wang H, Gillis A, Zhao C, Lee E, Wu J, et al. (2011) Crocidolite asbestosinduced signal pathway dysregulation in mesothelial cells. Mutat Res 723: 171176

11. Tooker BC, Newman LS, Bowler RP, Karjalainen A, Oksa P, et al. (2011) Proteomic detection of cancer in asbestosis patients using SELDI-TOF discovered serum protein biomarkers. Biomarkers 16: 181-191.

12. Kao SC, Griggs K, Lee K, Armstrong N, Clarke S, et al. (2011) Validation of a minimal panel of antibodies for the diagnosis of malignant pleural mesothelioma. Pathology 43: 313-317.

13. Creaney J, Dick IM, Yeoman D, Wong S, Robinson BW (2011) Auto-antibodies to $\beta-F 1-A T P a s e$ and vimentin in malignant mesothelioma. PLoS One 6: e26515.

14. Rodríguez Portal JA (2012) Asbestos-related disease: screening and diagnosis. Adv Clin Chem 57: 163-185.

15. Miao AJ, Wang WX (2004) Relationships between cell-specific growth rate and uptake rate of cadmium and zinc by a coastal diatom. Mar Ecol Prog Ser 275 103-113.

16. (2011) Asbestos Fibers and Other Elongate Mineral Particles: State of the Science and Roadmap for Research. DHHS (NIOSH) Publications \& Products.

17. Wegdam W, Moerland PD, Meijer D, de Jong SM, Hoefsloot HC, et al. (2012)
A critical assessment of SELDI-TOF-MS for biomarker discovery in serum and tissue of patients with an ovarian mass. Proteome Sci 10: 45

18. Tooker BC, Bowler RP, Orcutt JM, Maier LA, Christensen HM, et al. (2011) SELDI-TOF derived serum biomarkers failed to differentiate between patients with beryllium sensitisation and patients with chronic beryllium disease. Occup Environ Med 68: 759-764.

19. McLerran D, Grizzle WE, Feng Z, Bigbee WL, Banez LL, et al. (2008) Analytica validation of serum proteomic profiling for diagnosis of prostate cancer: sources of sample bias. Clin Chem 54: 44-52.

20. Hu J, Coombes KR, Morris JS, Baggerly KA (2005) The importance of experimental design in proteomic mass spectrometry experiments: some cautionary tales. Brief Funct Genomic Proteomic 3: 322-331.

21. Gemoll T, Roblick UJ, Auer G, Jörnvall H, Habermann JK (2010) SELDI-TOF serum proteomics and colorectal cancer: a current overview. Arch Physio Biochem 116: 188-196.

22. Callesen AK, Mogensen O, Jensen AK, Kruse TA, Martinussen T, et al. (2012) Reproducibility of mass spectrometry based protein profiles for diagnosis of ovarian cancer across clinical studies: A systematic review. J Proteomics 75 2758-2772.

23. Engwegen JY, Mehra N, Haanen JB, Bonfrer JM, Schellens JH, et al. (2007) Validation of SELDI-TOF MS serum protein profiles for renal cell carcinoma in new populations. Lab Invest 87: 161-172.

24. van Winden AW, Gast MC, Beijnen JH, Rutgers EJ, Grobbee DE, et al. (2009) Validation of previously identified serum biomarkers for breast cancer with SELDI-TOF MS: a case control study. BMC Med Genomics 2: 4.

25. Li J, Orlandi R, White CN, Rosenzweig J, Zhao J, et al. (2005) Independent validation of candidate breast cancer serum biomarkers identified by mass spectrometry. Clin Chem 51: 2229-2235.

26. Qin XJ, Ling BX (2012) Proteomic studies in breast cancer (Review). Onco Lett 1: 735-743.
This article was originally published in a special issue, Algorithm Evaluation and Validation in Proteomics handled by Editor(s). Dr. Tamanna Sultana, University of Pittsburgh, USA 